\title{
Antibiotic prophylaxis for preventing endophthalmitis after intravitreal injection: a systematic review
}

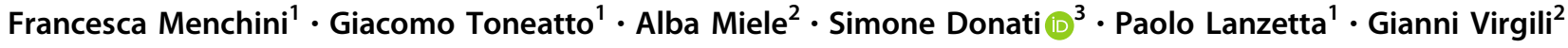

Received: 30 July 2017 / Revised: 26 December 2017 / Accepted: 19 February 2018 / Published online: 11 June 2018

(c) The Royal College of Ophthalmologists 2018

\begin{abstract}
Purpose To assess the effect of topical antibiotic prophylaxis on the rate of post-operative endophthalmitis after intravitreal injection (IVI).

Methods We conducted a systematic review of studies comparing the rates of endophthalmitis in eyes receiving IVI of different drugs with and without topical antibiotic prophylaxis, by searching MEDLINE and EMBASE up to June 2016. The methodological quality of the included studies was assessed using the Cochrane Risk of Bias tool and the Risk Of Bias in Non-randomized Studies of Interventions (ROBINS-I) for randomized clinical trials (RCTs) and non-randomized studies, respectively. We used a random-effects meta-analysis to compute the odds ratio (OR) of endophthalmitis with antibiotic prophylaxis compared with no prophylaxis and conducted subgroup analyses to compare the efficacy of different regimens and classes of antibiotics on endophthalmitis rates.

Results We identified 1 randomized and 12 non-randomized studies that reported 74 cases of endophthalmitis in 147,203 IVIs using antibiotic prophylaxis compared with 55 cases in 211,418 IVIs with no prophylaxis. The overall OR of endophthalmitis for antibiotic prophylaxis vs. no prophylaxis was 1.33 (95\% CI 0.75-2.38). Leave-one-out sensitivity analyses showed that the exclusion of the only study with a serious risk of bias significantly increased the risk of endophthalmitis in the antibiotic prophylaxis group compared with control (OR: 1.62, 95\% CI: 1.17, 2.34). There was no difference in the endophthalmitis rate associated with any other factor analyzed, including type of antibiotic, type of drug injected, or antibiotic prophylaxis regimen.

Conclusions Antibiotic prophylaxis does not reduce the rate of endophthalmitis following IVI and might potentially be associated with an increased risk of post-operative infection.
\end{abstract}

\section{Introduction}

The use of intravitreal injection (IVI) as a means of delivering intraocular therapeutic agents has grown

Electronic supplementary material The online version of this article (https://doi.org/10.1038/s41433-018-0138-8) contains supplementary material, which is available to authorized users.

Francesca Menchini

francescamenchini@gmail.com

1 Department of Medical and Biological Sciences-Ophthalmology, University of Udine, Udine, Italy

2 Department of Ophthalmology, University of Florence, A.O.U.C, Florence, Italy

3 Ophthalmology Clinic, Department of Medicine and Surgery, University of Insubria, Varese, Italy dramatically over the past decade, becoming the main treatment modality for several common ocular conditions, including exudative age-related macular degeneration, macular edema secondary to diabetes and retinal vein occlusion. The efficacy and the progressive broadening of indications of intravitreally injected inhibitors of vascular endothelial growth factors (VEGFs), and to a lesser extent steroids and more recently ocriplasmin, has made IVI the most performed procedure in ophthalmology [1].

The risk-benefit profile of IVI is good, with a low incidence of serious ocular adverse events, the most feared of which remains post-injection endophthalmitis because of its rapid course and potential evolution to severe vision loss. The reported incidence of endophthalmitis following IVI is low, ranging from 0.01 to $0.26 \%$ [2-16].

At first, investigational randomized clinical trials (RCTs) with anti-VEGF required the use of topical antibiotics before and after IVI in order to minimize the risk of post-IVI 
endophthalmitis, borrowing the habit from ocular incisional surgical procedures, such as cataract surgery and pars plana vitrectomy. Although topical antibiotics have been shown to reduce the conjunctival flora in patients receiving IVI, a substantial body of evidence suggests that repeated and shortterm ocular exposure to antibacterial eye drops significantly increases antibiotic resistance of ocular surface flora and may potentially lead to a higher risk of infection due to the selection of antibiotic-resistant bacterial strains [17-22].

The use of povidone-iodine on the ocular surface together with an accurate aseptic technique is the only procedure proven to reduce the risk of post-operative endophthalmitis in anterior segment surgery [23, 24].

Although some authors have suggested that topical povidone-iodine and antibiotics may act synergistically to decrease conjunctival flora [25], the results of an RCT did not show any additional benefit of the combination of these topical drugs [26].

Although the conflicting findings of these studies suggest that the effectiveness of antibiotic prophylaxis in preventing endophthalmitis after IVI remains unclear, many ophthalmologists still recommend prophylaxis with antibiotic eye drops in the peri-procedural period.

We conducted a systematic review of randomized and non-RCTs to assess the effect of peri-operative topical antibiotic prophylaxis on the risk of endophthalmitis following IVIs.

\section{Methods}

\section{Eligibility criteria}

We planned to include RCTs and non-randomized studies (NRS), which compared the rates of endophthalmitis in eyes receiving IVI of different drugs with no topical antibiotic prophylaxis versus eyes where topical antibiotic prophylaxis was administered either pre-operatively, postoperatively or both. We included studies in which patients underwent IVI for any reason (e.g., exudative age-related macular degeneration, macular edema secondary to diabetes and retinal vein occlusion, vitreo-macular traction syndrome), regardless of surgical technique, setting (office vs. operating room), type of drug, dosage or schedule. We excluded studies where IVI was performed in combination with an incisional surgical procedure, such as phacoemulsification or vitrectomy, due to the intrinsic risk of endophthalmitis with these procedures.

\section{Outcome measure}

The diagnosis of endophthalmitis is usually made when anterior segment and vitreous inflammation is confirmed biomicroscopically. Bacterial or fungal infections cannot always be detected by the culture of aqueous or vitreous samples. Our primary outcome measure was the occurrence of post-operative endophthalmitis after IVI as reported by study investigators, including cases of culture-positive and culture-negative endophthalmitis.

\section{Search strategy, study selection and data extraction}

We searched MEDLINE and EMBASE (January 1950 to June 2017) using the search strategy described in Appendix 1. We also hand-searched the reference lists of the included studies. Two authors independently reviewed the titles and abstracts for inclusion. Disagreements between the two review authors were resolved by a third author. For each included study, we extracted the number of endophthalmitis cases and the total number of injections in the antibiotic prophylaxis group and in the no prophylaxis group. We also recorded the type of prophylaxis regimen (preand post-operative), the class of antibiotic administered, and the type of intravitreal drug injected (anti-VEGF, triamcinolone acetonide (TA), dexamethasone implant, ocriplasmin).

\section{Methodological quality assessment}

We assessed the risk of bias of RCTS using the Cochrane Risk of Bias tool [27] and that of NRS using the Risk Of Bias of Non-randomized Studies of Interventions (ROBINS-I) tool [28].

\section{Data analysis}

Our unit of analysis was each IVI, because data on a patient basis are seldom reported and difficult to interpret. The odds ratio (OR) was our measure of effect. We did not aim to extract adjusted OR estimates because adjustment of confounders is typically not possible in studies of the occurrence of endophthalmitis, which is a rare adverse event.

Our primary analysis was standard random-effects metaanalysis using Stata 14.2 software (StataCorp, College Station, TX) [29]. We also displayed fixed-effects metaanalysis to assess the robustness of the assumptions made, as well as the effect of outlying studies using a leaveone-out meta-analytic technique. Sweeting et al. found that fixed-effects models performed well with sparse data [30].

Meta-regression was used to measure the effect of covariates on the action of antibiotics, specifically: fluoroquinolones vs. other antibiotics; post-injection antibiotic regimen vs. pre- and post-injection antibiotic regimen; and use of anti-VEGF alone vs. anti-VEGF and triamcinolone and other type of drugs. 
Finally, we further checked to what extent results were sensitive to assumptions regarding between-study heterogeneity using Bayesian methods [30], by adopting techniques that are reported in detail in a previous study on a similar topic [31]. In short, assumptions on priors on between-study standard deviation ranged from flat, uniformative priors (uniform distribution between 0 and 10) to a half-normal prior in which the median value was compatible with limited variation of ORs across studies, corresponding to a relative OR:2. These analyses were conducted using the software Winbugs 1.4 [29].

\section{Results}

\section{Results of search}

We retrieved 1253 titles and abstracts from MEDLINE, 1184 titles and abstracts from EMBASE and 1 additional abstract identified through other sources, of which we obtained and reviewed the full text for 68 . We excluded 55 non-comparative studies.

We included 13 studies in this review, consisting of 1 RCT [32], 12 NRS, [19, 33-41] of which 2 were cohort studies within an RCT [42, 43] (Fig. 1).

\section{Characteristics of included studies}

All studies aimed to assess the incidence of endophthalmitis after IVI of anti-VEGF or anti-VEGF and TA or antiVEGF, steroids and ocriplasmin and the effect of ocular prophylactic topical antimicrobial drugs on the incidence of endophthalmitis (Table 1). In NRS, assignment to antibiotic prophylaxis or no prophylaxis was performed according to three modalities: at surgeons' discretion, by center or by period. When allocation was by center, patients in one center were treated with antibiotics and patients in the other center received no prophylaxis. When treatment allocation was temporal, subjects included received prophylaxis over a defined period of time and did not thereafter. The centers included in the study by Casparis were an academic and a municipal hospitals in Switzerland; in Meyer's study both centers were university clinics in Germany. The authors state that the procedures were identical or very similar between the two institutions and we reasonably assumed that they were comparable in terms of population due to geographic localization.

We excluded the 2012 data in the study by $\mathrm{Li}$ et al. because the authors reported that the use of peri-IVI antibiotics was progressively discontinued, with the large majority of patients not using prophylaxis by January 2013, creating a mixed cohort of patients [38].

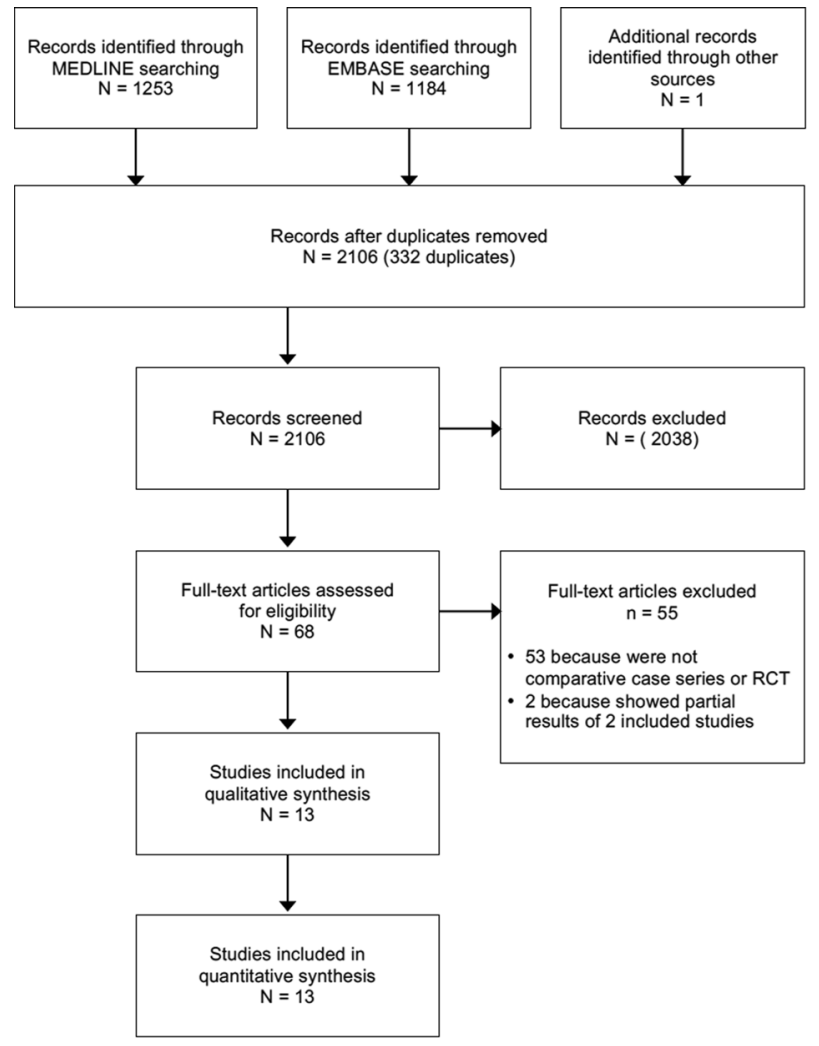

Fig. 1 Flow diagram of the study selection process

In the study by Bhavsar et al. [42], two endophthalmitis cases that occurred in 13 injections performed with topical antibiotic prophylaxis but without povidone-iodine due to a protocol deviation were excluded.

Regarding the type of prophylactic regimen employed, in nine of the included studies surgeons chose to use a post-IVI antibiotic prophylaxis regimen [19, 32-37, 39, 40]. In two studies, either pre- and post-IVI prophylaxis was used [38, 41]. Two studies reported results of three different antimicrobial regimens (pre-, post- and pre+post-IVI) [42, 43].

Type of prophylaxis was not specified in three studies [34, 42, 43]. Among the seven studies where the antimicrobial agent was described, fluoroquinolones was the class of choice in five studies [19, 32, 33, 35, 41], in three studies prophylaxis was mixed, performed using either fluoroquinolone or other different antibiotics [36, 38, 39], and in one study physicians prescribed aminoglycoside or macrolide eye drops [37].

Anti-VEGF drugs were administered in all the studies: six studies reported the incidence of endophthalmitis following anti-VEGF injections alone [32, 34-36, 39, 43], six studies following anti-VEGF and triamcinolone IVIs [19, 33, 37, 40-42], and one study following anti-VEGF, triamcinolone, dexamethasone implant and ocriplasmin IVIs [38]. 


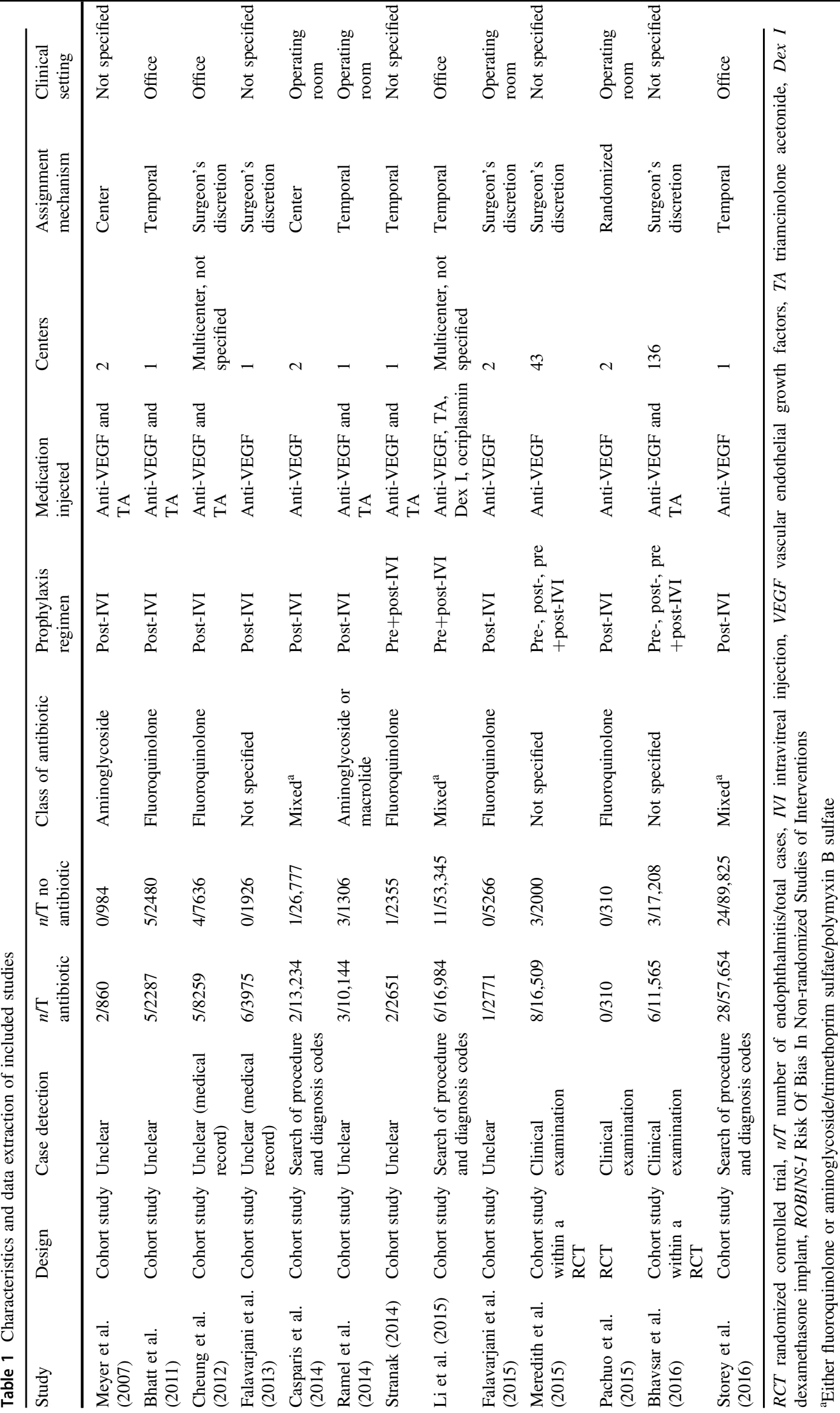


Fig. 2 Random-effect metaanalysis presenting the OR of endophthalmitis for any antibiotic prophylaxis vs control (upper diamond). Fixed-effect meta-analysis is also shown (lower diamond) and is used to present study weights in the forest plot. $\mathrm{M}-\mathrm{H}=$ Mantel-Haenszel (fixed-effects method for meta-analysis), D $+\mathrm{L}=$ Dersimonian and Laird (random-effects method for meta-analysis) for metaanalysis)

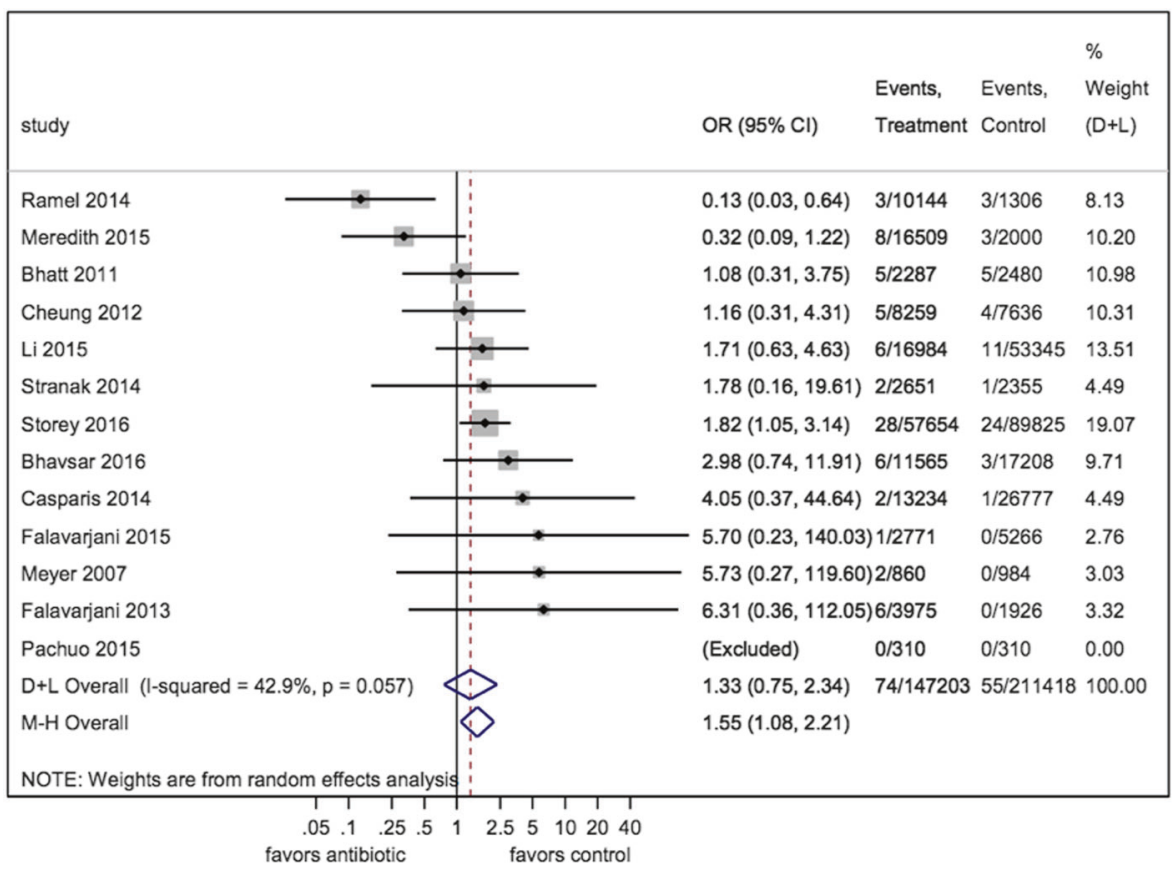

\section{Methodological quality of included studies}

The study by Pachuo et al., the only RCT included in this review, was assessed as at unclear risk of bias due to lack of information regarding all the domains of the Cochrane Collaboration's tool for assessing risk of bias [27], including sequence generation and allocation concealment [32].

When ROBINS-I was applied to NRS [28], the overall study quality was good, with little or no difference detected among studies in terms of bias due to confounding, selection of participants, missing data, measures of outcome and selection of the results (ROBINS-I table available online). For the topical antibiotic prophylaxis group, we assumed that patients' adherence to the assigned intervention was good, since the course of treatment was short and antibiotic eye drops are usually well tolerated. During the course of the studies by Storey and $\mathrm{Li}$, there was a transition phase where the use of antibiotic drops was not well defined, generating a mixed cohort of patients [38, 39]. Storey et al. did not include in the primary analysis any injections and endophthalmitis cases occurred during this period of time [39]. In contrast, data from this transitional phase were included by $\mathrm{Li}$ et al. [38], but the strict temporal definition of different study periods allowed us to identify the exact number of patients belonging to the mixed cohort and to exclude them during the data extraction process. All other studies adopted well-defined intervention as a short-course use of antibiotic eye drops.

We assumed that selection of cohorts in different periods of time and different centers did not influence study outcomes if co-intervention protocols were strictly observed.
We considered the use of povidone-iodine and eyelid speculum to be mandatory co-interventions.

In the study of Bhavsar et al. [42], two endophthalmitis cases occurred in 13 injections administered without povidone-iodine as required by the study protocol and therefore we excluded these data from the analysis.

Storey et al. reported that eyelid speculum was employed at physician discretion and we assumed that its use was likely to be balanced between the groups with or without antibiotics [39].

The retrospective case series by Ramel et al. [37] was assessed as at serious risk of bias since the injection protocol changed during the course of the study, making the two groups difficult to compare. Sterile lid speculum was abandoned and a disposable device for IVI, called InVitria, was adopted during the last 2 months in the antibiotic prophylaxis group and for the entire period, 4 months, in the group of patients who received no prophylaxis.

\section{Endophthalmitis after IVI with topical antibiotic prophylaxis versus no prophylaxis}

Figure 2 shows the number of endophthalmitis cases by presence or absence of topical antimicrobial prophylaxis together with type of prophylaxis regimen, class of antibiotics, type of drug injected. Seventy-four endophthalmitis cases were reported in 147,203 patients with antibiotic prophylaxis, compared with 55 cases in 211,418 patients with no prophylaxis. All studies reported at least one endophthalmitis case in each study arm, with the exception of Pachuo et al. [32], who recorded no events in either 
treatment or control groups. Three studies reported no events in the group that received no antibiotic prophylaxis $[34,35,40]$.

Our random-effects analysis of all 13 studies (Fig. 2) resulted in a pooled OR of 1.33 (95\% credible interval (CI): $0.75,2.34$ ) for the comparison between the group with antibiotic prophylaxis and the group with no prophylaxis. There was moderate between-study heterogeneity (Isquared $43 \%, p=0.057)$, meaning that effects varied importantly across studies.

This estimate suggests that the use of antibiotics increases the risk of endophthalmitis by $1 / 3$.

The fixed-effect estimate showed a significant increase in the risk of endophthalmitis in the treatment group by about 50\% (OR: 1.55 ; 95\% CI: 1.08, 2.21).

Leave-one-out sensitivity (Table 2) analyses showed that only the exclusion of Ramel turned the estimate to a statistically significant harm with antibiotics (OR: 1.62, $95 \%$ CI: $1.17,2.34$ ) with no heterogeneity (I-squared $0.3 \%)$ [37].

Meta-analyses of rare adverse events based on sparse data are often sensitive to assumptions regarding betweenstudy heterogeneity [30]. Following the approach reported in the Methods section, a Bayesian sensitivity analysis allowing for extreme heterogeneity obtained an OR: 1.62 (95\% CI: $0.79,3.94)$ and a probability that the effect of antibiotics may increase the risk of endophthalmitis (OR $>1$ ) of $92.3 \%$. This probability was $95.4 \%$ assuming very limited heterogeneity among studies, confirming the robustness of our meta-analyses.

Table 2 Leave-one-out sensitivity analyses showed that only the exclusion of Ramel (2014) turned the estimate to a significant harm with antibiotics (OR: 1.62, 95\% CI: $1.17,2.34)$ with no heterogeneity (I-squared $0.3 \%$ )

\begin{tabular}{llll}
\hline Study & OR $(95 \%$ CI $)$ & $p$-Value & I-squared \\
\hline All & $1.33(0.75,2.34)$ & 0.324 & $42.9 \%$ \\
Bhatt (2011) & $1.37(0.73,2.59)$ & 0.330 & $47.6 \%$ \\
Bhavsar (2016) & $1.22(0.66,2.25)$ & 0.521 & $44.8 \%$ \\
Casparis (2014) & $1.26(0.70,2.28)$ & 0.439 & $46.0 \%$ \\
Cheung (2012) & $1.36(0.72,2.56)$ & 0.342 & $47.8 \%$ \\
Falavarjani (2013) & $1.26(0.71,2.25)$ & 0.432 & $44.8 \%$ \\
Falavarjani (2015) & $1.28(0.71,2.28)$ & 0.411 & $46.0 \%$ \\
Li (2015) & $1.29(0.67,2.49)$ & 0.446 & $47.8 \%$ \\
Meredith (2015) & $1.55(0.92,2.62)$ & 0.100 & $28.4 \%$ \\
Meyer (2007) & $1.27(0.71,2.27)$ & 0.419 & $45.7 \%$ \\
Pachuo (2015) & $1.33(0.75,2.34)$ & 0.324 & $42.9 \%$ \\
Ramel (2014) & $\mathbf{1 . 6 2}(\mathbf{1 . 1 7}, \mathbf{2 . 3 4})$ & $\mathbf{0 . 0 1 1}$ & $\mathbf{0 . 3 \%}$ \\
Storey (2016) & $1.27(0.63,2.54)$ & 0.502 & $44.8 \%$ \\
Stranak (2014) & $1.32(0.72,2.40)$ & 0.372 & $48.0 \%$ \\
\hline
\end{tabular}

\section{Subgroups analyses}

Supplemental Figures 3-5 show meta-analyses by subgroups:

Type of antibiotic: fluoroquinolone vs. others of mixed (Supplemental Figure 3)

Type of drug injected: anti-VEGF vs. anti-VEGF+TA (Supplemental Figure 4)

Antibiotic prophylaxis regimen: post-IVI vs. pre-IVI or mixed (Supplemental Figure 5).

There was no difference in the endophthalmitis rate associated with any other factor analyzed, including type of antibiotic ( $p=0.626$ for fluoroquinolones vs. others), type of drug injected ( $p=0.688$ for anti-VEGF only vs. antiVEGF plus triamcinolone and others), or antibiotic prophylaxis regimen $(p=0.694$ for antibiotics post-IVI vs. pre and/or after).

\section{Discussion}

\section{Summary of results}

Our primary meta-analysis of 12 NRS and 1 RCT confirmed that the risk of endophthalmitis following IVI does not decrease with topical antibiotic prophylaxis.

The evidence supporting this finding was mostly based on good-quality NRS and was assessed by means of ROBINS-I, a recently developed methodological tool, which covers a broad spectrum of potential sources of bias [28]. Leave-one-out meta-analyses showed that the exclusion of a single study [37] at greater risk of bias, led to conclude that antibiotic prophylaxis might in fact increase the risk of endophtalmitis. Similar conclusions were reached when fixed-, rather than random-effects meta-analyses were conducted, as well as in Bayesian meta-analysis assuming limited between-study heterogeneity. Taken together, our meta-analyses confirm no benefit and potential harm with antibiotic prophylaxis.

This result can be explained by the finding that repeated use of topical antibiotic prophylaxis might induce the selection of antibiotic-resistant bacterial strains and increase the amount of multidrug-resistant organisms [18-22], even when administered for a short period of time and at low doses [44].

The results of our systematic review also support the recent guidelines of the American Academy of Ophthalmology that discourage the use of antibiotic prophylaxis for IVI $[45,46]$. 


\section{Comparison with other review}

A recently published systematic review by Benoist d'Azy et al. [47], also concluded that antibiotic prophylaxis is not required when performing IVI and that strict rules of asepsis remain the only evidence-based prophylaxis to avoid endophthalmitis. Our meta-analyses differ under many aspects from the study by Benoist d'Azy et al. regarding search strategy, eligibility criteria, data extraction and study quality assessments. Moreover, we identified four more studies, increased the number of events by about $50 \%$ and doubled the number of injections at the denominator, totaling over 350,000 IVIs in qualitative and quantitative analysis. Benoist d'Azy et al. used the Strengthening the Reporting of Observational Studies in Epidemiology (STROBE) tool to assess the accuracy and completeness of study reporting [48]. However, STROBE is not an appropriate instrument to evaluate the risk of bias of observational research.

Reibaldi et al. have recently published a meta-analysis of proportion of patient developing endophthalmitis in 60 studies [49]. These authors included all study designs, including non-comparative case series, and they rather used study arms of NRS as if they were independent to pool the frequency of endophtalmitis in studies with and without prophylaxis. They found a threefold increase in the risk of endophthalmitis with antibiotics, which is likely to be an overestimate of their adverse effect, because the comparative nature of NRS was not exploited.

\section{Strengths and limitations of this review}

We suggest that our review offers a number of improvements over previous work. We chose the new ROBINS-I tool to assess risk of bias of NRS because it addresses weaknesses not considered in previously available approaches [50], and includes key features such as specification of the target trial and effect of interest, and assessments of several signaling questions within seven bias domains. This led to identify a study at serious risk of bias with the variation of a major co-intervention during the last period, which made the two groups difficult to compare [37]. The potential difference between the lid speculum and the InVitria device has been identified by Dossarps et al., who demonstrated that the use of a disposable conjunctival fixed mold is associated with an increased incidence of endophthalmitis $(p=0.011)$ [51].

A limitation of our review is that we did not plan to perform separate analyses of culture-positive and culturenegative endophthalmitis. In fact, this complication is usually diagnosed on the basis of a spectrum of clinical features including pain, decreased visual acuity, hypopyon and posterior segment inflammation, and is frequently underestimated by culture tests [52].
The main limitation of the evidence supporting our conclusions is the moderate-high heterogeneity of ORs between studies. Heterogeneity is common in meta-analyses of observational studies because comparability of groups, as well as of outcome assessment, can be limited by unmeasured confounders and methodological issues. Such heterogeneity may suggest that there could be unknown sources of clinical variation on the occurrence of endophthalmitis [53]. Nonetheless, heterogenity was eliminated after a study at greater risk of bias was removed in leaveone-out meta-analyses.

Finally, the studies included in our review did not report estimates that adjusted for potential confounders, which is recommended in NRS meta-analyses. This should not be an important limitation, since endophthalmitis cannot be foreseen by patients and physicians, and we believe that selection bias is unlikely to arise with respect to antibiotic prophylaxis use.

In conclusion, our systematic review is the first large meta-analysis suggesting that peri-operative antibiotic prophylaxis is not only not required in IVIs, but can also be associated with an increased risk of post-operative endophthalmitis.

\section{Compliance with ethical standards}

Conflict of interest The authors declare that they have no conflict of interest.

\section{References}

1. Stalmans P, Benz MS, Gandorfer A, et al. Enzymatic vitreolysis with ocriplasmin for vitreomacular traction and macular holes. $\mathrm{N}$ Engl J Med. 2012;367:606-15.

2. Meyer $\mathrm{CH}$, Michels S, Rodrigues EB, et al. Incidence of rhegmatogenous retinal detachments after intravitreal antivascular endothelial factor injections. Acta Ophthalmol. 2011;89:70-75.

3. Meyer CH, Rodrigues EB, Michels S, et al. Incidence of damage to the crystalline lens during intravitreal injections. J Ocul Pharmacol Ther. 2010;26:491-5.

4. Brown DM, Kaiser PK, Michels M, ANCHOR Study Group. et al. Ranibizumab versus verteporfin for neovascular age related macular degeneration. N Engl J Med. 2006;355:1432-44.

5. Rosenfeld PJ, Brown DM, Heier JS, MARINA Study Group. et al. Ranibizumab for neovascular age-related macular degeneration. $\mathrm{N}$ Engl J Med. 2006;355:1419-31.

6. Brown DM, Campochiaro PA, Singh RP, et al. Ranibizumab for macular edema following central retinal vein occlusion: six month primary end point results of a phase III study. Ophthalmology. 2010;117:1124-33.

7. Campochiaro PA, Heier JS, Feiner L, et al. Ranibizumab for macular edema following branch retinal vein occlusion. Sixmonth primary end point results of a phase III study. Ophthalmology. 2010;117:1102-12.

8. Bhavsar AR, Googe JM Jr, Stockdale CR, et al. Risk of endophthalmitis after intravitreal drug injection when topical antibiotics are not required: the diabetic retinopathy clinical research network laser-ranibizumab-triamcinolone clinical trials. Arch Ophthalmol. 2009;127:1581-3. 
9. Brown DM, Nguyen QD, Marcus DM, et al. Long-term outcomes of ranibizumab therapy for diabetic macular edema: the 36-month results from two phase III trials: RISE and RIDE. Ophthalmology. 2013;120:2013-22.

10. Martin DF, Maguire MG, Fine SL, Comparison of Age related Macular Degeneration Treatments Trials (CATT) Research Group. et al. Ranibizumab and bevacizumab for treatment of neovascular age-related macular degeneration: two-year results. Ophthalmology. 2012;119:1388-98.

11. Heier JS, Brown DM, Chong V, et al. VIEW 1 and VIEW 2 Study Groups. Intravitreal aflibercept (VEGF trap-eye) in wet agerelated macular degeneration. Ophthalmology. 2012; 119:2537-48.

12. Moshfeghi AA, Rosenfeld PJ, Flynn HW Jr, et al. Endophthalmitis after intravitreal vascular [corrected] endothelial growth factor antagonists: a six-year experience at a university referral center. Retina. 2011;31:662-8.

13. Mason JO III, White MF, Feist RM, et al. Incidence of acute onset endophthalmitis following intravitreal bevacizumab (Avastin) injection. Retina. 2008;28:564-7.

14. Fung AE, Rosenfeld PJ, Reichel E. The International Intravitreal Bevacizumab Safety Survey: using the internet to assess drug safety worldwide. Br J Ophthalmol. 2006;90:1344-9.

15. Pilli S, Kotsolis A, Spaide RF, et al. Endophthalmitis associated with intravitreal anti-vascular endothelial growth factor therapy injections in an office setting. Am $\mathbf{J}$ Ophthalmol. 2008;145:879-82.

16. Fintak DR, Shah GK, Blinder KJ, et al. Incidence of endophthalmitis related to intravitreal injection of bevacizumab and ranibizumab. Retina. 2008;28:1395-9.

17. De Caro JJ, Ta CN, Ho HV, et al. Bacterial contamination of ocular surface and needles in patients undergoing intravitreal injections. Retina. 2008;28:877-83.

18. Storey P, Dollin M, Pitcher J, Post-Injection Endophthalmitis Study Team. et al. The role of topical antibiotic prophylaxis to prevent endophthalmitis after intravitreal injection. Ophthalmology. 2014;121:283-9.

19. Cheung CS, Wong AW, Lui A, et al. Incidence of endophthalmitis and use of antibiotic prophylaxis after intravitreal injections. Ophthalmology. 2012;119:1609-14.

20. Bhavsar AR, Stockdale CR, Ferris FL 3rd, et al. Diabetic Retinopathy Clinical Research Network. Update on risk of endophthalmitis after intravitreal drug injections and potential impact of elimination of topical antibiotics. Arch Ophthalmol. 2012;130:809-10.

21. Kim SJ, Toma HS, Midha NK, et al. Antibiotic resistance of conjunctiva and nasopharynx evaluation study: a prospective study of patients undergoing intravitreal injections. Ophthalmology. 2010;117:2372-8.

22. Milder E, Vander J, Shah C, et al. Changes in antibiotic resistance patterns of conjunctival flora due to repeated use of topical antibiotics after intravitreal injection. Ophthalmology. 2012; 119:1420-4.

23. Ciulla TA, Starr MB, Masket S. Bacterial endophthalmitis prophylaxis for cataract surgery: an evidence-based update. Ophthalmology. 2002;109:13-24.

24. Speaker MG, Menikoff JA. Prophylaxis of endophthalmitis with topical povidone-iodine. Ophthalmology. 1991;98:1769-75.

25. Ta CN, Egbert PR, Singh K, et al. Prospective randomized comparison of 3-day versus 1-hour preoperative ofloxacin prophylaxis for cataract surgery. Ophthalmology. 2002; 109:2036-40.

26. Moss JM, Sanislo SR, Ta CN. A prospective randomized evaluation of topical gatifloxacin on conjunctival flora in patients undergoing intravitreal injections. Ophthalmology. 2009;116:1498-501.
27. Higgins JP, Altman DG, Cochrane Statistical Methods Group, Cochrane Bias Methods Group, eds. Chapter 8: Assessing risk of bias in included studies. In: Higgins JP, Green S, editors. Cochrane Handbook of Systematic Reviews of Interventions. Version 5.0.0. Part 2. 2008. Available at: http://www.mrcbsu.cam. ac.uk/cochrane/handbook500/chapter_8/8_assessing_risk_of_bia s_in_included_studies.htm. Accessed 19 March 2012.

28. Sterne JAC, Hernán MA, Reeves BC, et al. ROBINS-I: a tool for assessing risk of bias in non-randomized studies of interventions. BMJ. 2016;355:i4919.

29. Spiegelhalter DJ, Thomas A, Best NG. WinBUGS Version 1.4 User Manual. Cambridge: MRC Biostatistics Unit; 2012. Available at http://www.mrc-bsu.cam.ac.uk/bugs/winbugs/contents. shtml Accessed 19 March 19

30. Sweeting MJ, Sutton AJ, Lambert PC. What to add to nothing? Use and avoidance of continuity corrections in meta-analysis of sparse data. Stat Med. 2004;23:1351-75.

31. Govetto A, Virgili G, Menchini F, et al. A systematic review of endophthalmitis after microincisional versus 20 -gauge vitrectomy. Ophthalmology. 2013;120:2286-91.

32. Pachuo MA, Sahto AA, Quraishi, et al. Role of post-injection antibiotics after intravitreal bevacizumab injection in preventing endophthalmitis. Pak J Ophthalmol. 2015;31:44-47.

33. Bhatt SS, Stepien KE, Joshi K. Prophylactic antibiotic use after intravitreal injection. Effect on endophthalmitis rate. Retina. 2011;31:2032-6.

34. Falavarjani KG, Modarres $\mathrm{M}$, Hashemi $\mathrm{M}$, et al. Incidence of acute endophthalmitis after intravitreal bevacizumab injection in a single clinical center. Retina. 2013;33:971-4.

35. Falavarjani KG, Aghamirsalim M, Modarres M, et al. Endophthalmitis after resident-performed intravitreal bevacizumab injection. Can J Ophthalmol. 2015;50:33-36.

36. Casparis $\mathrm{H}$, Wolfensberger TJ, Becker $\mathrm{M}$, et al. Incidence of presumed endophthalmitis after intravitreal injection performed in the operating room. A retrospective multicenter study. Retina. 2014;34:12-17.

37. Ramel JC, Bron AM, Isaico R, et al. Incidence of endophthalmitis after intravitreal injection: is antibioprophylaxis mandatory? J Fr Ophtalmol. 2014;37:273-9.

38. Li AL, Wykoff CC, Wang R, et al. Endophthalmitis after intravitreal injection. Role of prophylactic topical ophthalmic antibiotics. Retina. 2015;0:1-8.

39. Storey P, Dollin M, Rayess N, et al. The effect of prophylactic topical antibiotics on bacterial resistance patterns in endophthalmitis following intravitreal injection. Graefes Arch Clin Exp Ophthalmol. 2016;254:235-42.

40. Meyer CH, Mennel S, Eter N. Endophthalmitis rate mit und ohne topische post-operative antibiotikagabe nach intravitrealer Avastin-lnjektion. Ophthalmologe. 2007;104:952-57.

41. Stranak Z, Veith M, Studeny P, et al. The incidence of endophtalmitis after the application of intravitreal injections in FNKV with regard to various prophylactic antibiotic regimens. Ceskà a Slov Oftalmol. 2014;70:184-8.

42. Bhavsar AR, Glassman AR, Stockdale CR, et al. for the Diabetic Retinopathy Clinical Research Network. Elimination of topical antibiotics for intravitreous injections and the importance of using povidone-iodine. Update from the Diabetic Retinopathy Clinical Research Network. JAMA Ophthalmol. 2016; 134:1181-1183.

43. Meredith TA, McCannel CA, Barr C, et al. Postinjection endophthalmitis in the comparison of age-related macular degeneration treatments trials (CATT). Ophthalmology. 2015;122:817-21.

44. Mino De Kaspar H, Hoepfner AS, Engelbert M, et al. Antibiotic resistance pattern and visual outcome in experimentally-induced 
Staphylococcus epidermidis endophthalmitis in a rabbit model. Ophthalmology. 2001;108:470-8.

45. American Academy of Ophthalmology. How to Give Intravitreal Injections. External link https://www.aao.org/eyenet/article/howto-give-intravitreal-injections. Released 04.2013.

46. American Academy of Ophthalmology. Five Things Physicians and Patients Should Question. External link http://www. choosingwisely.org/societies/american-academy-of-ophtha lmology/; Released 21.02.2013.

47. Benoist d'Azy C, Pereira B, Neughton G, et al. Antibioprophylaxis in prevention of andophthalmitis in intravitreal injection: a systematic review and meta-analysis. PLoS One. 2016;11:e0156431.

48. Von Elm E, Altman DG, Egger M, et al. STROBE initiative. Strengthening the Reporting of Observational Studies in Epidemiology (STROBE) statement: guidelines for reporting observational studies. BMJ. 2007;335:806-8.

49. Reibaldi M, Pulvirenti A, Avitabile T, Bonfiglio V, Russo A, Mariotti C, et al. Pooled estimates of incidence of endophthalmitis after intravitreal injection of anti-vascular endothelial growth factor agents with and without topical antibiotic prophylaxis. Retina. 2017;38:1-11. https://doi.org/10.1097/IAE.000000000 0001583.

50. Sanderson S, Tatt ID, Higgins JPT. Tools for assessing quality and susceptibility to bias in observational studies in epidemiology: a systematic review and annotated bibliography. Int J Epidemiol. 2007;36:666-76.

51. Dossarps D, Bron AM, Koehrer P, et al. Endophthalmitis after intravitreal injections: incidence, presentation, management, and visual outcome. Am J Ophthalmol. 2015;160:17-25.

52. Pongsachareonnont $\mathrm{P}$, Honglertnapakul W, Chatsuwan $\mathrm{T}$. Comparison of methods for identifying causative bacgterial microorganisms in presumed acute endophthalmitis: conventional culture, blood culture, and PCR. BMC Infect Dis. 2017; 17:165.

53. Park Y, Kim KS, Park YH. Acute endophthalmitis after intravitreal injection and preventive effect of preoperative topical antibiotics. J Ocul Pharmacol Ther. 2013;29:900-5. 